\title{
Hypercube Simulation of Electric Fish Potentials
}

\author{
Roy Williams, Brian Rasnow and Christopher Assad
}

\author{
California Institute of Technology \\ Pasadena, California
}

\begin{abstract}
We present a simulation of the electrosensory input of the weakly electric fish Apteronotus leptorhynchus. This fish senses its environment by producing a sinusoidal voltage difference between its body and tail sections, causing an electric field and a current distribution in the surrounding water. If an object is nearby which has different electrical conductivity from the surrounding water, the current distribution is disturbed on the skin of the fish. The fish senses this difference from the usual current distribution, and infers the presence and location of the object.

Mathematically, the problem is to solve a potential equation in the domain exterior to the fish with Cauchy boundary conditions, in the presence of an induced dipole arising
\end{abstract}

from the object, and extract the potential difference across the fish skin.

We have created an unstructured triangular mesh covering the two-dimensional manifold of the fish skin, using the Distributed Irregular Mesh Environment (DIME), then used the Boundary Element Method to solve for the potential derivative at the fish skin.

The computational problem is the solution of a full set of simultaneous linear equations, where there is an equation for each node of the boundary mesh, typically about 100 - 200. We have used an NCUBE hypercube to calculate the matrix elements and solve these equations, once for each relative position of the fish and the test object. We present some early results from the simulation. 


\section{Biological Background}

All animals are faced with the computationally intense task of continuously acquiring and analyzing sensory data from their environment. To ensure maximally useful data, animals appear to use a variety of motor strategies or behaviors to optimally position their sensory apparatus. In all higher animals, neural structures which process both sensory and motor information are likely to exist which can coordinate this exploratory behavior for the sake of sensory acquisition. We believe the cerebellum may be involved in this motor-sensory loop.

To study this possibility, we have chosen the weakly electric fish, which use a unique electrically based means of exploring their environment ${ }^{1,2}$. These nocturnal fish, found in murky waters of the Congo and Amazon, have developed electrosensory systems to allow them to detect objects without relying on vision. In fact, in some species this electric sense appears to be their primary sensory modality.

This sensory system relies on an electric organ which generates a weak electric field surrounding the fish's body that in turn is detected by specialized electroreceptor cells in the fish's skin. The presence of animate or inanimate objects in the local environment causes distortions of this electric field, which are interpreted by the fish. In some species of weakly electric fish, the electric organ fires a short pulse and then is silent, in effect gating the electrosensory information into the nervous system at discrete times rather than entering as a continuous stream like most other sensory modalities. Other species sample their environment with a pulse in the frequency domain, ie. by generating a nearly sinusoidal electrical discharge. The simplicity of the sensory signal, in addition to the distributed external representation of the detecting apparatus, makes the weakly electric fish an excellent animal with which to study the involvement in sensory discrimination of the motor system in general and body position in particular.

It is of value experimentally and also interesting to note that some of these fish have the largest cerebellum, relative to their brain and body mass, of any class of animals. The experiments we have undertaken are specifically aimed at understanding to what extent the exploratory behavior of the fish involves coordinated positioning of both its electric organ and its electroreceptors to resolve objects in its local electric field.

Simulations in two dimensions $\mathrm{s}^{3,4}$ and our measurements with actual fish have shown that body position, especially the tail angle, significantly alter the fields near the fish's skin. We are currently developing freeze-frame video techniques to be used in combination with high resolution electrode arrays positioned in the fish tank to record fish behavior in response to a variety of environmental stimuli.

To study quantitatively how the fish's behavior affects the "electric images" of objects, we are developing threedimensional computer simulations of the electric fields that the fish generate and detect. These simulations, when calibrated with the measured fields, should allow us to identify and focus on behaviors that are most relevant to the fish's sensory acquisition tasks, and to predict the electrical consequences of the behavior of the fish with higher spatial resolution than possible in the tank.

Being able to visualize the electric fields, in false color on a simulated fish's body as it swims, may provide a new level of intuition into how these curious animals sense and respond to their world.

In this paper, we discuss a physical model of an electric fish, then the equivalent mathematical problem, which is a solution of Laplace's equation in the region exterior to the fish and the object it is sensing. We give a brief description of the Boundary Element method for solving this problem, and explain why this method is well suited for a distributed parallel architecture. Finally we describe some early results from the simulation.

\section{Physical Model}

We need to reduce the great complexity of a biological organism to a manageable physical model. The ingredients of this model are the fish body, shown in Figure 1, the object that the fish is sensing, and the water exterior to both the fish and the object.

The real fish has some projecting fins, and our first approximation is to neglect these because their electrical properties are essentially the same as those of water.

Our second approximation is to simplify the timedependence of the electric field set up by the fish. The time constant associated with electric field variations in a dielectric medium is of order dielectric constant divided by conductivity ${ }^{5}$. For water this charactaristic time is measured in fractions of a microsecond, and for a perfectly conducting object is zero. The time between pulses of the electric organ is about a millisecond in $A$. leptorhynchus, so that if the fish is sensing a perfectly conducting object, it is safe to ignore time variation and model the fields as static. For some plant materials, however, this time constant may be large, and the fish may sense phase information (analogously to humans using the phase difference between the ears to sense the 
direction of a sound).

In this paper, we shall concentrate on the statis approximation. There is thus an electric field, maintained by the fish, which causes a current flow proportional to the electric field according to Ohm's law.

We will assume that the fish is exploring a small conductive object, such as a small metal sphere. First we reduce the geometrical aspect of the object to being pointlike, yet retaining some relevant electrical properties. Except when the object is another electric fish, we expect the object to have no active electrical properties, but only to be an induced dipole, so that in the presence of an electric field the object becomes a dipole of strength proportional to the field and oriented opposite to the field. The proportionality constant is the polarizability of the object.

Thus the polarizability is the only parameter describing the object. In this first paper, we shall not attempt to calibrate experimental measurements and computed results, but merely estimate this parameter. Polarizability has the dimensions of volume, so we shall model an object of polarizability $1 \mathrm{~cm}^{3}$, since this is the size of object used in the experiments.

We now come to the modelling of the fish body itself. This consists of a skin with electroreceptor cells which can detect potential difference, and a rather complex internal structure. We shall assume that the source voltage is maintained at the interface between the internal structure and the skin, so that we need not be

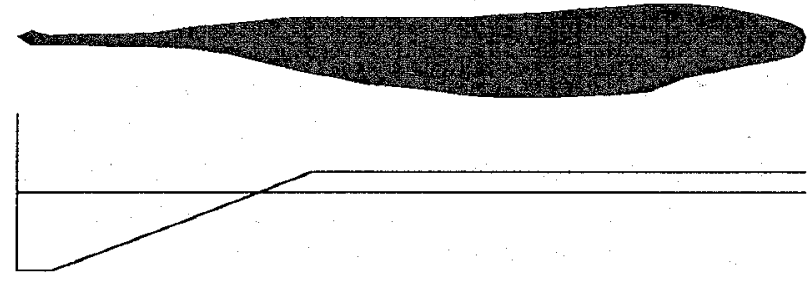

Figure 1. Top, top view of the fish Apteronotus leptorhynchus, Middle, side view of the fish. The fish is about $20 \mathrm{~cm}$ long.Bottom, modelled voltage profile $\phi$ along the interior of the fish, from $-100 \mathrm{mV}$ at the tail with a linear ramp to $+25 \mathrm{mV}$ at the head. The fins and tail are not shown.

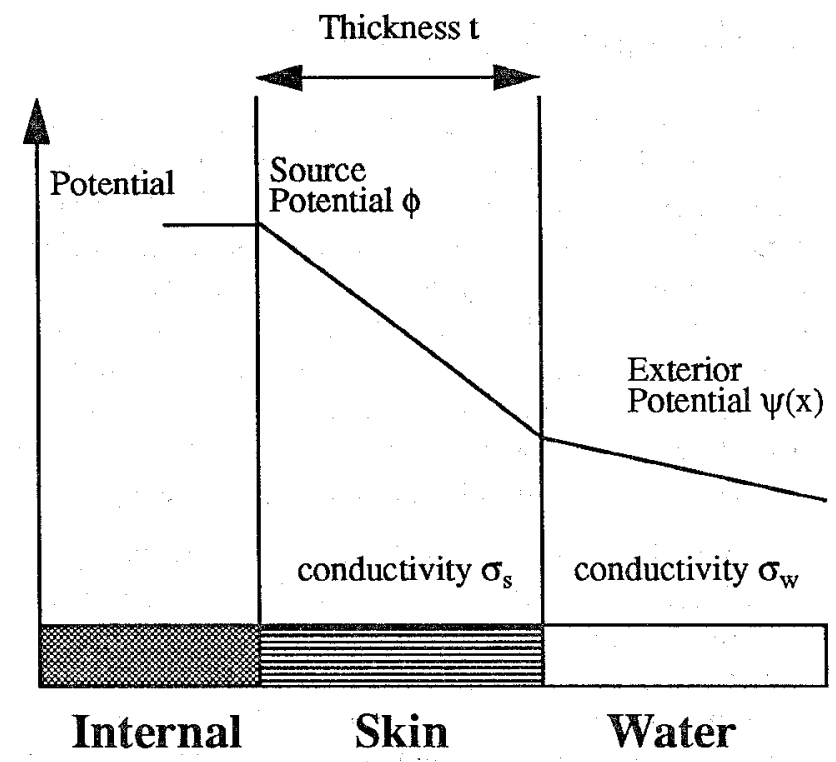

Figure 2: A section through the fish skin, with electrical potential plotted vertically. The potential is assumed linear within the skin.

concerned with the details of the internal structure. Thus the fish body is modelled as two parts: an internal part with a given voltage distribution on its surface, surrounded by a skin with variable conductivity.

Because of the voltage on the internal body, a current distribution is set up in the fish skin and water, which have different conductivities. The signal from the electroreceptor cells in the skin is assumed to depend on the potential difference across the skin ${ }^{6}$.

We shall simplify the model a little more by assuming that the skin thickness is small compared to the size of the fish. This is not equivalent to neglecting the skin altogether, since it is the combination of skin thickness and conductivity which determines its electrical properties; the zero-skin-thickness approximation merely removes geometrical complexity from the model in exchange for a slightly more complex boundary condition at the surface of the fish body, as discussed below.

Figure 2 shows a section through the body of the fish, with a graph of the voltage or potential superimposed. We define $\phi$ to be the potential at the interface between the fish skin and the internal part of the fish, and the scalar field $\psi(x)$ to be the potential field in the water exterior to the skin. The conductivities of the skin and water are written $\sigma_{\mathrm{s}}$ and $\sigma_{\mathrm{w}}$ respectively.

We write the normal derivative of the exterior potential as $\psi_{\mathrm{n}}$, and conservation of current then implies that the 
slope of the potential in the skin be $\psi_{\mathrm{n}} \sigma_{\mathrm{w}} / \sigma_{\mathrm{s}}$. We shall now assume that the potential varies linearly from the inside to the outside of the skin; sufficient justification for this would be that either the skin is thin compared to the body thickness, or that the source potential varies slowly over the skin compared to the skin thickness.

Using the thickness $t$ of the skin, we find the boundary condition

$$
\psi-\xi \Psi_{n}=\phi
$$

where the effective skin thickness $\xi$ is defined to be

$$
\xi=t \frac{\sigma_{w}}{\sigma_{s}}
$$

This is a Cauchy or mixed boundary condition for the exterior potential.

Conservation of charge is again the guiding physical law to obtain the differential equation satisfied by $\psi$ in the water. Mathematically, it means that the divergence of the current density is zero; thus we can use Ohm's law to write

$$
\nabla \cdot(\sigma \nabla \psi)=0
$$

where $\sigma$ is the conductivity of the water, assumed uniform, and $\nabla \psi$ is the electric field. This equation reduces to $\nabla^{2} \psi=0$, Laplace's equation.

\section{Mathematical Theory}

The Boundary Element method ${ }^{7,8}$ has been used for many applications where it is necessary to solve a linear elliptic partial differential equation. The derivation is particularly simple for the case of Laplace's equation, which we present with less than complete mathematical rigor.

Green's theorem states that if functions $U$ and $V$ are free of singularities in a domain $\Omega$, with the normal outward from $\Omega$, then

$$
\int_{\Omega}\left(U \nabla^{2} V-V \nabla^{2} U\right) d^{3} q=\int_{\partial \Omega}\left(U \frac{\partial V}{\partial n}-V \frac{\partial U}{\partial n}\right) d^{2} q
$$

We define $V$ to be the desired solution $\psi$, and for some fixed point $p$, we set

$$
U(q)=\frac{1}{|p-q|}
$$

Since $\nabla^{2} \psi=0$ and $\nabla^{2} U=-4 \pi \delta(p-q)$, Green's theorem becomes

$$
\psi(p) A^{\Omega}(p)=\int_{\partial \Omega}\left(\frac{1}{|p-q|} \frac{\partial}{\partial n_{q}} \psi-\psi \frac{\partial}{\partial n_{q}|p-q|}\right)
$$

where $A^{\Omega}(p)$ is the solid angle around $p$ subtended by $\Omega$; for example if $\Omega$ is a cube, then $A$ is $4 \pi$ inside the cube, $2 \pi$ on a face, $\pi$ on an edge, and $\pi / 2$ at a corner of the cube.

We can simplify the notation by introducing linear operators $B^{\Omega}$ and $C^{\Omega}$, which can be defined by their actions on a dummy function $u$ :

$$
\begin{aligned}
& \left(B^{\Omega} u\right)(x)=\int_{\partial \Omega} u\left(x^{\prime}\right) \frac{\partial}{\partial n^{\prime}} \frac{1}{\left|x-x^{\prime}\right|} d x^{\prime} \\
& \left(C^{\Omega} u\right)(x)=-\int_{\partial \Omega} u\left(x^{\prime}\right) \frac{1}{\left|x-x^{\prime}\right|} d x^{\prime}
\end{aligned}
$$

so that the Boundary Element Theorem for Laplace's equation becomes

$$
A^{\Omega} \psi+B^{\Omega} \psi+C^{\Omega} \psi_{n}=0
$$

Notice that if $\Omega^{\prime}=\mathfrak{R}^{3} \backslash \Omega$, which is the region outside $\Omega$, then $A^{\Omega}+A^{\Omega^{\prime}}=4 \pi, B^{\Omega}+B^{\Omega^{\prime}}=0$, and $C^{\Omega}=C^{\Omega^{\prime}}$.

When the function $\psi$ is approximated with Finite Elements as discussed below, the operators $A, B$ and $C$ become matrices, with $A$ diagonal.

The Boundary Element theorem (2) provides a relation between $\psi$ and its normal derivative at any point on the surface of $\Omega$, so that given another relation between the two (the boundary condition (1)), we can solve for both. We wish to solve for the normal derivative of the potential, so we combine the Boundary Element theorem and the boundary condition to obtain

$$
(\xi A+\xi B+C) \psi_{n}=-(A+B) \phi
$$

Note that this result is only true if the domain $\Omega$ is free of singularities.

In the case of our model of the fish, the domain of interest is that outside the fish and the object, extending to infinity. We have solved for the normal derivative because it is this that determines the potential difference across the fish skin, which in turn determines the response of the electroreceptor cells.

The solution of (3) yields the potential derivative for the fish with no object in its environment. The solution for the fish with object is obtained by introducing an induced dipole. Let $\Psi$ be the potential in the presence of the dipole. Without loss of generality, we may assume the dipole to be at the origin, so that the vector strength $\mathbf{d}$ of 
the dipole is (proportional to) the gradient of $\psi$ at the origin. This gradient may be written as a surface integral by differentiating the Boundary Element theorem:

$$
\mathrm{d} \propto \nabla \psi(0)=-\int_{\partial \Omega}\left(\frac{\mathbf{q}}{q^{3}} \frac{\partial}{\partial n_{q}} \psi-\psi \frac{\partial}{\partial n_{q}} \frac{q}{q^{3}}\right) d^{2} q
$$

We now separate out the singular part of $\Psi$, defining $\Psi_{1}$ by subtracting the dipole contribution:

$$
\Psi_{1}=\Psi-\frac{\mathbf{d} \cdot \mathbf{r}}{r^{3}}
$$

Given that $\Psi_{1}$ satisfies the Boundary Element equation (2), because it is free of singularities, and $\Psi$ satisfies the boundary conditions, we may derive the equation satisfied by $\Psi$ :

$$
(\xi A+\xi B+C) \Psi_{n}=-(A+B)\left(\phi-\frac{\mathbf{d} \cdot \mathbf{r}}{r^{3}}\right)+C \frac{\partial}{\partial n} \frac{\mathbf{d} \cdot \mathbf{r}}{r^{3}}
$$

\section{Computational Method}

In order to discretize the boundary element method, we have created a mesh of triangles covering the surface of the fish, as shown in Figure 3, using the Distributed Irregular Mesh Environment (DIME) ${ }^{9}$, a portable programming environment designed for calculations with unstructured triangular meshes on distributed memory parallel processors.

We discretize the field with linear Finite Elements:

$$
\Psi(x)=\sum_{\nu} \Psi_{v} N_{v}(x)
$$

where $\psi_{v}$ is the value of the field at the node $v$ and $N_{v}(x)$ is the piecewise linear function which is unity at the node $v$ and zero at every other node. The normal derivative can be similarly discretized.

As observed above, the operators $B$ and $C$ become matrices, and we define the matrix element $B_{\mu v}$ to be the value of,

$$
\left(B^{\Omega} N_{\mathrm{v}}\right)\left(x_{\mu}\right)
$$

which is the operator $B$ applied to the nodal basis function for node $v$ and evaluated at the position of node $\mu$. Similarly for the operator $C$.

We can calculate these matrix elements either by Gaussian integration ${ }^{10}$ on the triangles neighboring node $v$, or analytically. It is a useful check on the matrix
Figure 3: A typical mesh covering the surface of the fish, containing 190 nodes. The mesh is double-sheeted, for the two sides of the fish.

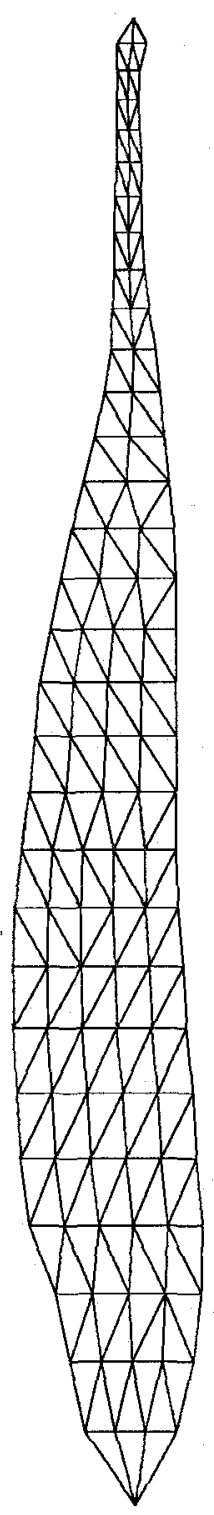

element calculation that as the number of Gauss points increases, the result approaches the analytic result.

To solve for the potential in the presence of the object, the procedure is then as follows. First we solve for the potential $\psi$ on the surface of the fish in the absence of the dipole singularity using (3), then calculate the dipole strength as the gradient of this potential at the position of the object using (4). Now we solve for $\Psi$ with this dipole, using equation (5). One way to visualize the result is to display $\Psi_{n}-\Psi_{n}$, which is proportional to the voltage difference acros the skin, and thus contains all the electrosensory information regarding the object which is 
accessible to the fish. Notice that it is the same matrix to be solved for both of these calculations, with different right hand sides. Thus it would be computationally efficient to decompose the matrix and back-substitute for each solve, rather than starting afresh each time.

For distributed memory parallel computation, we have a packaged LU solver ${ }^{11}$ for full matrices with partial pivoting, and the solver is used in three stages as follows. First the user makes an initialization call, sending the matrix size; then an LU decomposition call, where the user passes a function pointer which will calculate any required matrix element; then a back-substitution stage, where the user passes a function pointer which will calculate an element of the right-hand-side vector.

The manipulation of the mesh is done redundantly in each processor, so that before the solve step each processor has an identical copy of the mesh, and is thus capable of calculating any of the matrix elements or right-hand-side elements. When the solver is initialized, the parallel decomposition of the matrix is dealt with by the solver; and it automatically balances the matrix element computation and solving between the processors without user input.

The solution vector is returned in a distributed form to the processors, and a simple combining operation across the parallel machine gives the complete solution to each processor. We may visualize the solution using a variety of the tools from the DIME environment.

This code is an example of distributed memory programming at its easiest and most efficient: the difficult part of the programming is the sequential part, which is setting up and manipulating the mesh over the fish skin, and the most time-consuming part of the computation is the setting up and solution of linear equations, which happens without any effort from the user. The parallel programming has been done in writing the matrix solver: when more such tools are available, parallel programming will become much easier.

Let us compare the Boundary Element method with a more conventional finite difference approach to solving elliptic problems.

To implement the finite difference method, we would first make a mesh filling the domain of the problem, that is a three dimensional mesh, then for each mesh point set up a linear equation relating its field value to that of its neighbors. We would then need to solve a set of sparse linear equations. In the case of an exterior problem such as ours, we would need to pay special attention to the farfield, making sure the mesh extends out far enough and that the proper approximation is made at this outer boundary.
With the Boundary Element method, we discretize only the surface of the domain, and again solve a set of linear equations, except that now they are no longer sparse. The far-field is no longer a problem, since this is taken care of analytically.

If it is possible to make a regular grid surrounding the domain of interest, then the Finite Difference method is probably more efficient, since multigrid methods or alternating direction methods will be faster than the solution of a full matrix. It is with complex geometries however, that the Boundary Element method can be faster and more efficient, on sequential or distributed memory machines. It is much easier to produce a mesh covering a curved two-dimensional manifold than a three-dimensional mesh filling the space exterior to the manifold. If the manifold is changing from step to step, the 2D mesh need only be distorted, whereas a 3D mesh must be completely remade, or at least strongly smoothed, to prevent tangling. If the 3D mesh is not regular, the user faces the not inconsiderable challenge of explicit load balancing and communication at the processor boundaries.

We feel that the existence of distributed matrix solving software makes the Boundary Element method preferable to conventional Finite Difference methods, since it is competitive in computation time, and much easier to program.

\section{Results}

Figure 4 shows four fish in various unlikely positions.

For this initial investigation we have chosen to set the effective skin thickness $\xi$ to be $2 \mathrm{~cm}$, after measurements by Scheich and Bullock ${ }^{12}$; this figure has significant error, and of course the real fish has variable $\xi$ over its body.

Figure 5 shows a side view of the fish with the free field $\psi$ (no object) shown in gray scale, and we can see how the potential ramp at the skin-body interface has been smoothed out by the resistivity of the skin. Figure 6 shows the computed potential contours for the midplane around the fish body, showing the dipole field emanating from the electric organ in the tail.

Figure 7 shows the difference field $\Psi_{n}-\psi_{n}$ for three object positions, near the tail (left), at the center (middle) and near the head of the fish (right). In each case the object is $3 \mathrm{~cm}$ above the midplane, and the fish is $21 \mathrm{~cm}$ long. It can be seen that the difference field, which is also the sensory input for the fish, is greatest when the object is close to the head. A better view of the difference voltage is shown in Figure 8, which shows the values of 
the difference voltage on the midline of the fish, for various object positions. Again it may be seen that the maximum sensory input occurs when the object is close to the head of the fish, rather than the tail, from which the dipole field emanates.

\section{References}

1. T. H. Bullock and W. Heiligenberg, (eds), Electroreception, Wiley, New York, 1986.

2. H. W. Lissman, On the Function and Evolution of Electric Organs in Fish, J. Exp. Biol., 35 (1958) 156.

3. W. Heiligenberg, Theoretical and Experimental Approaches to Spatial Aspects of Electrolocation, J. Comp. Physiol., 103 (1975).

4. M. Bacher, A New Method for the Simulation of Electric Fields, Generated by Electric Fish, and their Distortions by Objects, Biol. Cybern. 47 (1983) 51.

5. J. D. Jackson, Classical Electrodynamics, Wiley, New York, 1975, p. 296.

6. J. Bastian, Electrolocation, J. Comp. Physiol., 144 (1981)

7. T. A. Cruse and F. J. Rizzo (eds.), Boundary Integral Equation Method: Computational Applications in Applied Mechanics, ASME Proc. AMD-Vol. 11 (1975).

8. C. A. Brebbia et al., (eds), Boundary Elements, Springer-Verlag, Berlin, 1983.

9. R. D. Williams, DIME: A Users Manual, Caltech Concurrent Computation Project Report C3P-861 (1990).

10. R. W. Cowper, Gaussian Quadrature Formulas for Triangles, Int. J. Numer. Methods Eng, 7 (1973) 405.

11. P. G. Hipes, Comparison of $L U$ and Gauss-Jordan System Solvers for Distributed Memory Multiprocessors, Caltech Concurrent Computation Project report C3P-652c, To Be Published in Concurrency, Practice and Experience.

12. H. Scheich and T. H. Bullock, The Detection of Electric Fields from Electric Organs, in Electroreceptors and Other Specialized Receptors in Lower Vertebrates, (A. Fesand, ed.), Springer-Verlag, Berlin, 1974.

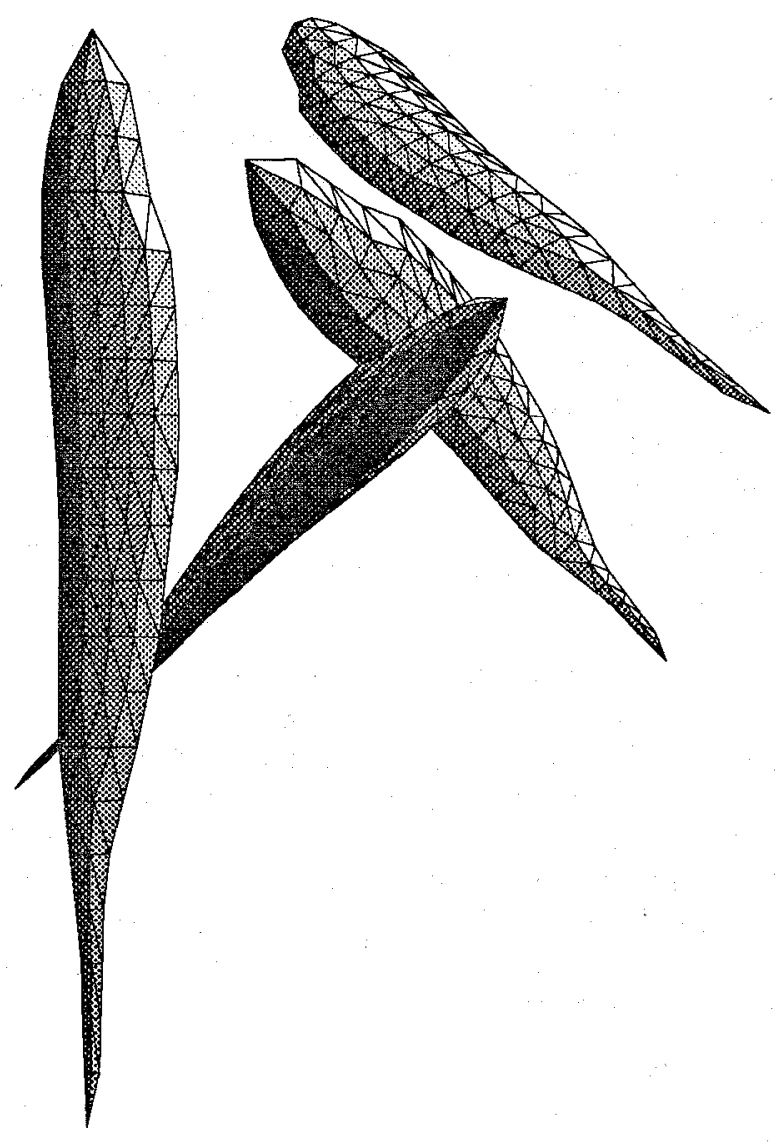

Figure 4: Four fish with simple shading. 


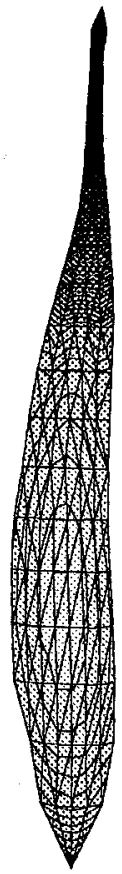

Figure 5: Potential distribution on the surface of the fish, with no external object.
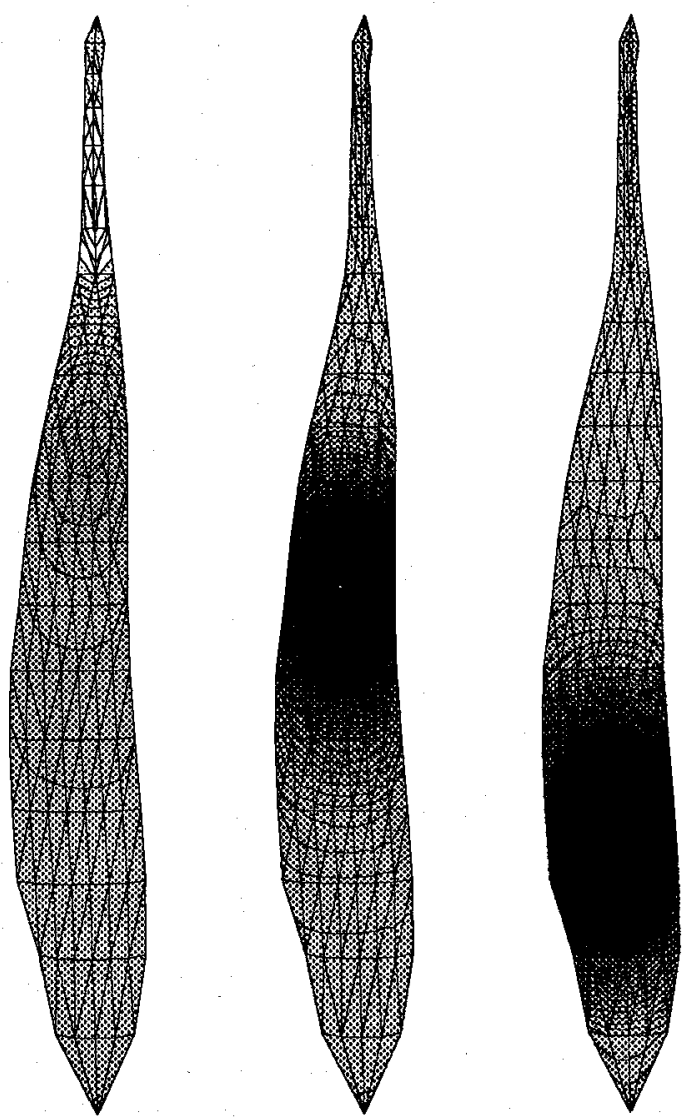

Figure 7: Gray-scale plots of voltage differences due to an object at positions (left) near tail, (middle) at center and (right) near head. Each object is $3 \mathrm{~cm}$ above midplane.

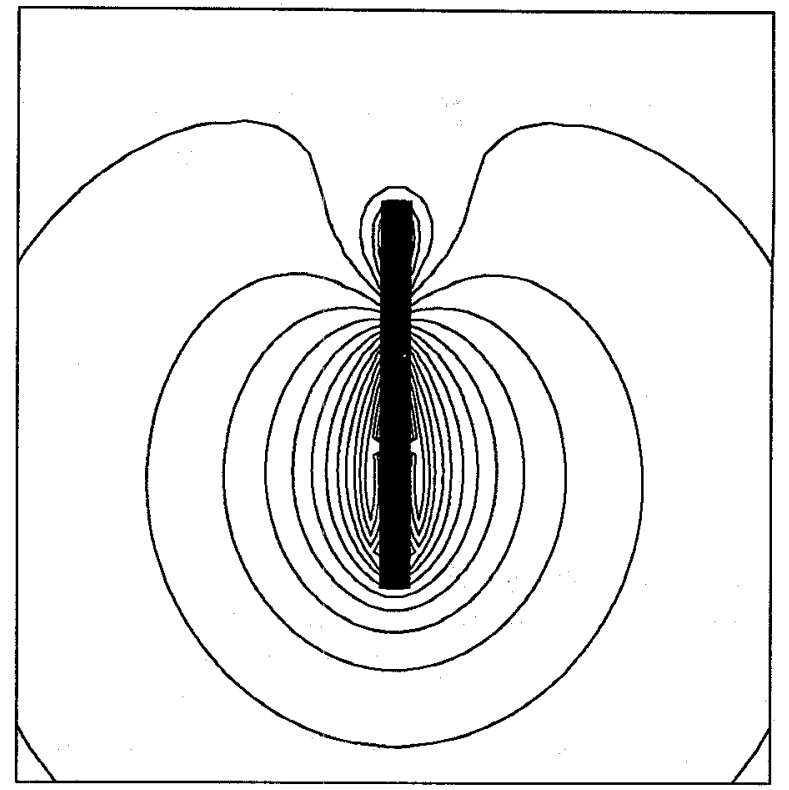

Figure 6: Potential contours on the midplane of the fish, showing dipole distribution from the tail.

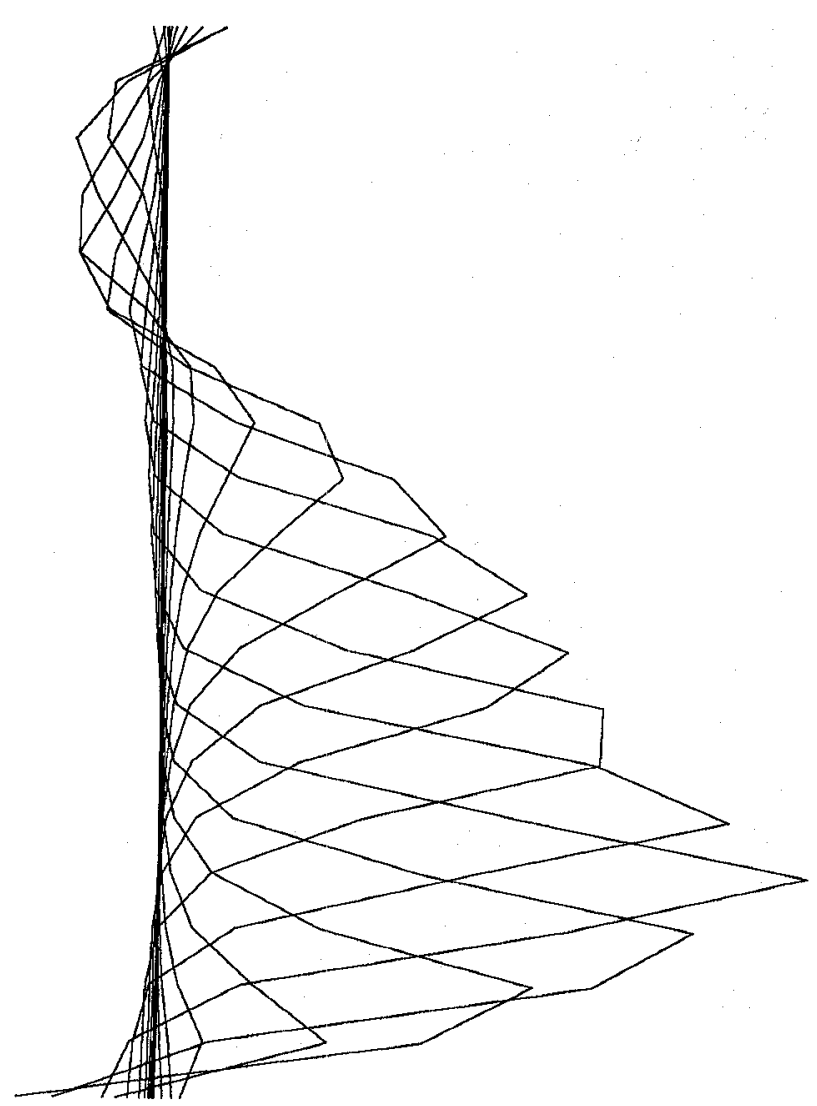

Figure 8: Envelope of voltage differences along midline of the fish, for 20 object postions, each $3 \mathrm{~cm}$ above midplane. 